\title{
Investigating the Lipid Selectivity of the Ammonium Transporter AmtB in Heterogeneous Nanodiscs
}

\author{
James E. Keener ${ }^{1}$ and Michael T. Marty1,2, \\ ${ }^{1}$ Department of Chemistry and Biochemistry and ${ }^{2}$ Bio5 Institute, University of Arizona, Tucson, AZ 85721, USA \\ KEYWORDS: Membrane proteins, lipids, nanodiscs, native mass spectrometry, detergent
}

\begin{abstract}
The structure and function of membrane proteins can be significantly impacted by the surrounding lipid environment, but membrane protein-lipid interactions in lipid bilayers are often difficult to study due to their transient and polydisperse nature. Here, we used two native mass spectrometry (MS) approaches to investigate how the Escherichia coli ammonium transporter (AmtB) selectively remodels its local lipid environment in heterogeneous lipoprotein nanodiscs. First, we used gas-phase ejection to isolate AmtB with bound lipids from heterogeneous nanodiscs with different combinations of lipids. Second, we used solution-phase detergent "flash" extraction as an orthogonal approach to study AmtB remodeling with native MS. Flash extraction of AmtB showed that Triton X-100 retains lipid selectivity, but C8E4 distorts preferential lipid interactions. Both approaches reveal that AmtB has a few tight binding sites for PC, is selective for binding PG overall, and is nonselective for PE, providing a detailed picture of how AmtB binds different lipid head groups in the context of mixed lipid bilayers.
\end{abstract}

\section{INTRODUCTION}

Membrane proteins play crucial roles in cellular processes and represent the majority of drug targets. $.^{1-3} \mathrm{Mem}-$ brane protein structure and function can be heavily influenced by interactions with the lipid environment, either by global bilayer properties or through direct lipid interactions. ${ }^{4-5}$ This interplay between proteins and lipids drives membrane organization and structure. For example, membrane proteins can be recruited to membrane microdomains based on local bilayer properties like curvature or thickness. ${ }^{6-7}$ Conversely, membrane proteins can remodel their local lipid environment by selectively binding different lipid types. ${ }^{8-9}$

Molecular dynamics simulations have provided significant insights about lipid remodeling, ${ }^{10}$ but membrane protein-lipid interactions remain challenging to study experimentally due to their dynamic and heterogeneous nature. ${ }^{11}$ Cryo-EM and X-ray crystallography provide high-resolution snapshots of stable membrane protein-lipid complexes with non-annular lipids. But, these structural methods are less suitable for annular lipids that dynamically exchange with bulk lipids in the surrounding bilayer.

Native mass spectrometry (MS) has been used to quantify, distinguish, and identify different kinds of bound lipids surrounding membrane proteins, ${ }^{12-15}$ but these experiments are generally limited to probing a small number of lipids in detergent micelles. To investigate a wider range of lipid interactions, we previously used nanodiscs, discoidal lipid bilayers encircled by two membrane scaffold protein (MSP) belts, to study membrane proteins in lipid bilayers with a larger number of bound lipids. However, past studies have only explored a single lipid or pair of lipids. ${ }^{16-17}$
Here, our goal was to investigate how membrane proteins remodel their surrounding lipid bilayer in more complex mixed lipid environments. We chose the ammonium transporter trimer (AmtB) from Escherichia coli (E. coli) because lipid interactions with this protein affect its stability and function. Specifically, AmtB binds and is stabilized by phosphatidylglycerol (PG) lipids. ${ }^{8}$ Furthermore, molecular dynamics simulations and activity assays revealed that $A m t B$ requires PG lipids for proper function. ${ }^{18}$

We performed native MS of AmtB-lipid complexes following gas-phase ejection or solution-phase detergent "flash" extraction of AmtB from mixed lipid nanodiscs composed of binary and ternary mixtures of palmitoyl-oleoyl-phosphatidylethanolamine (POPE), palmitoyl-oleoyl-phosphatidylcholine (POPC), and palmitoyl-oleoyl-phosphatidylglycerol (POPG). Ejection of AmtB revealed an overall selectivity for POPG, a few tightly bound POPC lipids, and no selectivity of POPE. Detergent flash extraction using Triton X-100 showed similar selectivity trends, but C8E4 extraction showed little to no lipid selectivity, illustrating that detergents can alter membrane protein-lipid interactions.

\section{METHODS}

Protein Expression and Purification. HIS-MBP-TEVAmtB and MSP1E3D1 were expressed in E. coli and purified as previously described. ${ }^{16,}{ }^{19}$ Briefly, AmtB was detergent extracted overnight with n-dodecyl- $\beta$-d-maltopyranoside (DDM, Anatrace) and n-octyl- $\beta$-d-glucopyranoside (OG, Anatrace) following isolation of membranes by ultracentrifugation. AmtB was then purified by immobilized affinity chromatography (IMAC), followed by size exclusion chromatography (SEC) on a HiLoad 16/600 Superdex 200 pg 

available under aCC-BY-NC-ND 4.0 International license.

(Cytiva) with $0.025 \%$ DDM. MSP1E3D1 was purified by IMAC using established protocols. ${ }^{20}$

Nanodisc Assembly and Purification. AmtB nanodiscs were assembled using MSP1E3D1(-), with the polyhistidine tag removed by TEV protease, and purified as previously described.16-17, 19 1-Palmitoyl-2-oleoyl-sn-glycero-3-phosphocholine (POPC), 1-palmitoyl-2-oleoyl-sn-glycero-3phospho-(1'-rac-glycerol) (POPG), and 1-palmitoyl-2oleoyl-sn-glycero-3-phosphoethanolamine (POPE) lipids from Avanti Polar Lipids were dissolved in chloroform and quantified by phosphate analysis. Lipids in chloroform were mixed to a molar ratio of 1:1 or 1:1:1, dried overnight, and resuspended in $100 \mathrm{mM}$ sodium cholate (Sigma Aldrich) to a final lipid concentration of $50 \mathrm{mM}$. Lipids, MSP, cholate, and $A m t B$ were mixed and incubated on ice for approximately 1 hour. Following addition of Amberlite XAD-2 beads (Sigma Aldrich), the reconstitution mixture was incubated at $4{ }^{\circ} \mathrm{C}$ overnight on an orbital shaker. AmtB nanodiscs were purified by IMAC followed by SEC using a Superose 6 10/300 Increase GL (Cytiva). Nanodiscs were incubated with TEV protease at $4{ }^{\circ} \mathrm{C}$ overnight to cleave the HISMBP tag. Nanodiscs were purified by another round of IMAC and SEC, and peak fractions were concentrated to 1-5 $\mu \mathrm{M}$ nanodisc. Samples were either analyzed immediately or flash frozen at $-80^{\circ} \mathrm{C}$ for storage. Error bars correspond to three replicate nanodisc assemblies that were prepared separately for each lipid composition.

Native MS of AmtB Nanodiscs. For ejection-based native MS, AmtB nanodiscs were gently mixed 19:1 v/v with neat glycerol carbonate at $>90 \%$ purity (Tokyo Chemical Industry Co., Inc.).16-17 The mixture was incubated for several minutes at room temperature prior to native MS.

For flash extraction, stock C8E4 (Anatrace) and Triton X100 (Sigma Aldrich) solutions were prepared by diluting to $20 \times$ the critical micelle concentration (CMC) in water. AmtB nanodiscs were gently mixed 9:1 v/v with stock detergent solutions to yield a final detergent concentration of $2 \times \mathrm{CMC}$, incubated on ice for 3 minutes, and then analyzed by native MS. Compared to gas-phase ejection (Figures S-1 and S-2), solution-phase detergent extraction yielded AmtB trimer that was much more stable with less subunit dissociation (Figures S-3, S-4, and S-5). Trimer dissociation was minimal for Triton X-100 and absent for C8E4. We also observed that Triton X-100 preserved a larger number of bound lipids, demonstrating that different detergents can be used to isolate different numbers of bound lipids.

Native MS of AmtB nanodiscs was performed as previously described ${ }^{16}$ using a Q-Exactive HF Orbitrap mass spectrometer (Thermo Fisher Scientific, Bremen) equipped with Ultra-High Mass Range modifications. ${ }^{21}$ Nano-electrospray ionization was performed in positive ion mode using borosilicate needles pulled with a P-1000 micropipette puller (Sutter Instrument, Novato, $\mathrm{CA}$ ). Samples were analyzed with a range of $2,000-30,000 \mathrm{~m} / \mathrm{z}$ at a resolution setting of 15,000 . Collision voltage was applied in the HCD cell and increased from 0 to $200 \mathrm{~V}$ in $20 \mathrm{~V}$ increments at 1- or 2minute acquisitions for each step. Important instrument settings included: $1.1-1.5 \mathrm{kV}$ capillary voltage, $200^{\circ} \mathrm{C}$ capillary temperature, and a trapping gas pressure of 7 . Nanodiscs with Triton X-100 were analyzed with a trapping gas pressure of 10 when the membrane scaffold protein (MSP) signal was relatively high. Ternary nanodiscs with glycerol carbonate were analyzed with a capillary temperature of $300{ }^{\circ} \mathrm{C}$ to aid with desolvation. Moreover, ternary and $50 \%$ POPE:POPG nanodiscs with glycerol carbonate were analyzed with $0-50 \mathrm{~V}$ of source fragmentation to aid with desolvation. Data for AmtB ejected from 50\% POPC:POPG nanodiscs was collected and described previously. ${ }^{17}$

Native MS Data Analysis. Analysis of native MS data was performed as previously described ${ }^{17}$ with slight modifications. MS data was analyzed using both UniDec ${ }^{22}$ and MetaUniDec. ${ }^{23}$ Important deconvolution parameters were as follows: mass range of 29-200 kDa, charge range of 1-25, mass sampled every $1 \mathrm{Da}$, and a peak full width at half maximum of 2.5 Th using a Gaussian peak shape function. Mass smoothing was used with mass differences corresponding to average lipid masses of 754.5 $\mathrm{Da}, 733.5 \mathrm{Da}, 739 \mathrm{Da}$, and $742.4 \mathrm{Da}$, for POPC:POPG, POPG:POPE, POPC:POPE, and POPG:POPC:POPE nanodiscs, respectively. For reference, the masses of the pure lipids are POPC $760 \mathrm{Da}$, POPG 749 $\mathrm{Da}$, and POPE $718 \mathrm{Da}$. The charge, point, and mass smooth widths were all set to 1 .

Lipids bound to proteins can undergo preferential gasphase dissociation at high levels of activation that is related to the gas-phase basicities of the headgroups. ${ }^{24}$ To avoid these gas-phase artifacts, we chose voltage ranges with lower levels of activation. The lowest voltage for each lipid mixture was set to where the peaks were first well-resolved. The maximum voltage for each lipid mixture was set to capture low numbers of bound lipids without observing significant artifacts or dissociation of the protein complex. For ejection-based native MS, the voltage ranges for POPC:POPE, POPG:POPE, POPC:POPG, and POPG:POPC:POPE were $60-160 \mathrm{~V}, 0-160 \mathrm{~V}, 60-200 \mathrm{~V}$, and 20-180 V, respectively. For detergent flash extraction, the voltage ranges for POPC:POPE, POPG:POPE, POPC:POPG, and POPG:POPC:POPE were 100-160 V, 100-160 V, 100$200 \mathrm{~V}$, and $100-180 \mathrm{~V}$, respectively. We did not observe lipid enrichment in line with the predicted gas-phase basicity of headgroups at these voltages, suggesting that we have avoided activation artifacts.

Spectra collected over these voltage ranges were deconvolved to zero-charge mass spectra and averaged to provide a comprehensive span of different membrane protein-lipid complexes. We extracted the center of mass for each peak from the deconvolved mass distribution corresponding to an AmtB-lipid complex. Only intensities above $50 \%$ of the maximum around each peak were used for extraction to minimize distortions from the baseline or noise. Following subtraction of the AmtB mass, we divided by the number of bound lipids to calculate the average lipid mass for a given number of bound lipids.

We observed small differences in the mass of AmtB, likely due to adduction or errors in mass accuracy, and corrected for this using a linear regression of number of bound lipids versus the center of mass for the peaks. Similar to our previous study, ${ }^{17}$ the correction was typically less than the mass of one water or ammonium molecule. Additionally, the average masses of bound lipids are not reported for 1 and 2 bound lipids, because average mass uncertainties are larger at lower numbers of bound lipids. Different peaks were present at different collision voltages, so the overall average mass for each peak was calculated by averaging each collision voltage step weighted by the squared intensity at that 

available under aCC-BY-NC-ND 4.0 International license.

voltage. The number of lipids bound to AmtB was somewhat different between lipid compositions and between gasphase and solution-phase extraction methods. To facilitate more direct comparison, the average lipid masses were plotted against the minimum common number of lipids observed for either binary (19 lipids) or ternary (14 lipids) mixtures.

\section{RESULTS AND DISCUSSION}

Ejection of AmtB from Two-Component Lipid Nanodiscs Reveals Lipid Enrichment. To investigate how AmtB selectively remodels its surrounding lipid environment, we used native MS to eject AmtB-lipid complexes from nanodiscs with binary mixtures of POPC, POPG, and POPE. For brevity, we refer to each lipid by its headgroup abbreviation: PC, PG, and PE. AmtB was assembled into nanodiscs with 50\% PC:PE, 50\% PG:PE, and 50\% PC:PG. The nanodiscs were analyzed by native MS using glycerol carbonate as a supercharging reagent to facilitate ejection of AmtB from the nanodisc. ${ }^{16}$ Collisional activation was gradually applied to eject AmtB with bound lipids (Figure 1A), and data were deconvolved to zero-charge mass spectra. Representative raw and zero-charge mass spectra are shown for each lipid mixture (Figures 1B, 1C, 1D, S-1), where each peak corresponds to AmtB with a specific number of bound lipids. We measured the average mass of bound lipids for each peak by subtracting the mass of AmtB and dividing the difference by the number of bound lipids. If there were no enrichment, the average lipid mass would be equal to the average of the two lipids. Any shifts in the average lipid masses indicate preferential binding of either the lighter or heavier lipid.

We first performed native MS of AmtB-lipid complexes ejected from 50\% PC:PE nanodiscs. Most bound lipids showed no enrichment, with average lipid masses almost exactly as expected for a 50:50 mixture of PC:PE. However, despite their similar chemical structures, the five lipids that were most tightly retained during ejection of AmtB had higher than expected average lipid masses, indicating slight enrichment in PC (Figure 1B). Thus, AmtB was not generally selective for PC over PE, except for a few tightly bound lipids.

To further examine the lipid selectivity of AmtB, we ejected it from 50\% PG:PE nanodiscs. Here, lipids bound to AmtB revealed around a 2:1 enrichment in PG over PE, which was consistent across all 19 lipid-bound states studied (Figure 1C). For example, the composition for 10 bound
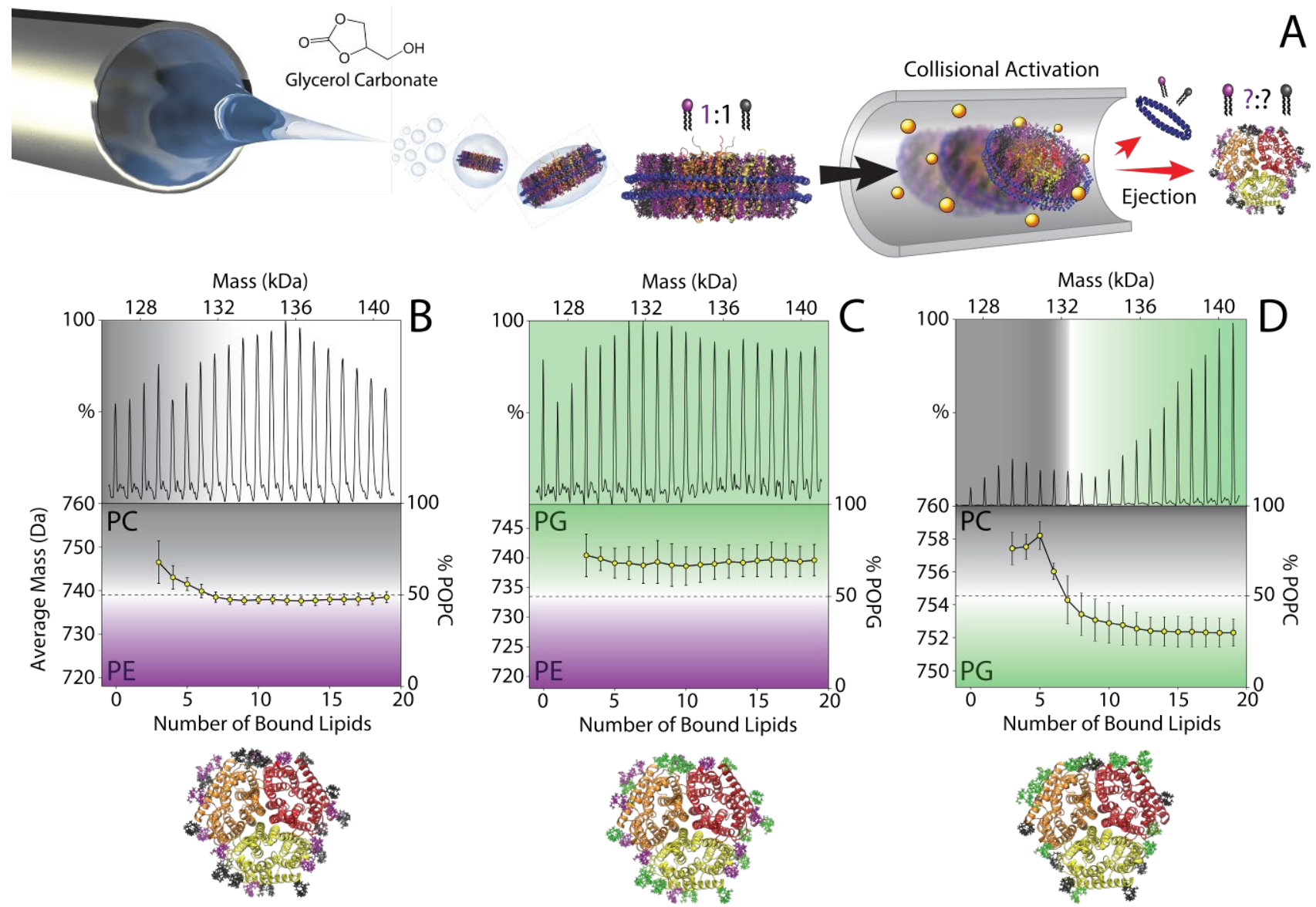

Figure 1. (A) Schematic for ejection of AmtB-lipid complexes from 50\% PC:PE nanodiscs during native MS. Summed deconvolved mass spectra (top) of AmtB-lipid complexes ejected from (B) 50\% PC:PE nanodiscs, (C) 50\% PG:PE nanodiscs, and (D) $50 \%$ PC:PG nanodiscs. The corresponding average masses of bound lipids are indicated below. Black, green, and purple regions represent enrichment in PC, PG, and PE, respectively. White regions represent no lipid enrichment. The initial expected average lipid masses corresponding to $50 \%$ lipid mixtures are indicated by dashed lines. Average masses heavier or lighter than the dashed line show lipid enrichment. Figure 1D adapted from ref. 17. Copyright 2020 American Chemical Society. 
lipids was $66 / 34 \pm 10 \% \mathrm{PG} / \mathrm{PE}$. Thus, AmtB remodels mixed PG:PE bilayers to selectively bind PG lipids in a roughly 2:1 ratio.

After observing consistent PG enrichment and slight PC enrichment for a few tightly bound lipids, we compared the lipid enrichment with previous data from AmtB-lipid complexes ejected from 50\% PC:PG nanodiscs (Figure 1D). Here, AmtB had distinct enrichment for two subsets of lipidbound states. The first six lipids that bound tightest showed significant enrichment of PC. Conversely, the next 13 bound lipids showed significant enrichment of PG. Specifically, the bound lipid composition for AmtB bound to 3 and 19 lipids was $77 / 23 \pm 9 \% \mathrm{PC} / \mathrm{PG}$ and $30 / 70 \pm 8 \% \mathrm{PC} / \mathrm{PG}$, respectively.

Collectively, these datasets reveal that AmtB selectively remodels its local lipid environment in two different ways. First, the most tightly bound lipids were enriched in PC, showing higher enrichment against PG but still detectable enrichment over PE. PE lipids only differ from PC lipids by three methyl groups, so these surprising results suggest that PC selectivity may be due to a few very specific binding interactions. Interestingly, PC binding has been shown to enhance the binding of $A m t B$ to the regulatory protein, $\mathrm{GlnK},{ }^{25}$ so these PC binding sites may affect AmtB interactions. Second, AmtB was significantly enriched in PG in both PG:PE and PC:PG mixtures, agreeing with previously established functional and stabilizing roles of $\mathrm{PG}$ for AmtB. ${ }^{8,18}$ No selective enrichment of PE was observed for either lipid mixture, so PE seems to play the role of a generic neutral lipid that participates non-selectively in the annular belt.

Ejection of AmtB from Three-Component Lipid Nanodiscs Confirms PC Selectivity. To probe lipid enrichment in a more complex lipid environment, we ejected AmtB from nanodiscs with a ternary mixture of all three lipids. Because there are three different mass components, we unfortunately cannot calculate an exact lipid composition from the average lipid mass. However, we can make predictions based on the binary data above and test whether the ternary data is consistent with the expected masses.

The average masses of most bound lipids were consistent with somewhere between no enrichment (a 1:1:1 mixture) and a 2:1 PG enrichment (2:1:1 PG:PC:PE) (Figure 2). Thus, we can rule out significant PE or PC enrichment, which would significantly alter the average lipid masses. These data are consistent with overall enrichment of PG in ternary nanodiscs, but we cannot confidently measure the degree of enrichment. Interestingly, the few most tightly bound lipids showed a gradual shift to heavier masses, supporting a likely enrichment of PC observed in binary data. Overall, the data from AmtB in ternary nanodiscs is consistent with AmtB being selective for PC for a few tightly bound lipids and selective for PG overall. However, the PC enrichment appears less substantial than in PC:PG nanodiscs, suggesting that the presence of PE dampens the lipid selectivity of AmtB in ternary mixtures.

Detergent Flash Extraction for Orthogonal Characterization of Lipid Selectivity. Ejection of AmtB-lipid complexes from heterogeneous nanodiscs allows us to probe selective lipid remodeling for a wide range of lipid-bound states, ${ }^{17}$ but it can be hard to relate the most tightly retained lipids upon gas-phase ejection with the most tightly bound lipids in solution. Recent studies from Prell and coworkers ${ }^{24}$ propose that different lipid head groups can dissociate differently at high levels of collisional activation due to differences in gas-phase basicity. Although we did not observe lipid compositions expected from this type of gas-phase dissociation, different gas-phase binding strengths could bias which lipids are retained.

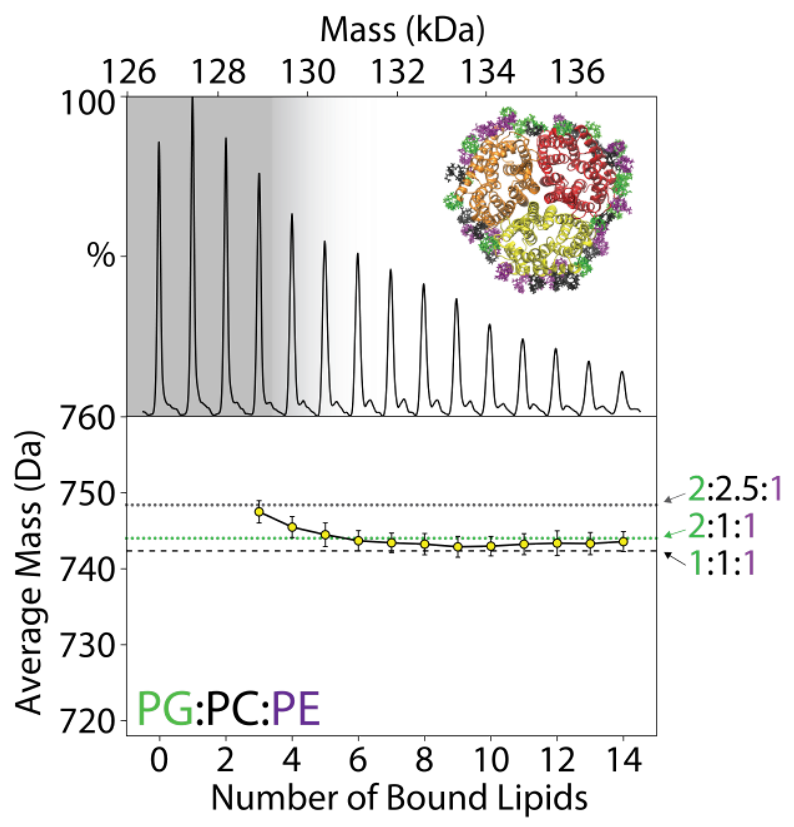

Figure 2. Summed deconvolved mass spectrum of AmtBlipid complexes ejected from 1:1:1 PG:PC:PE nanodiscs. The corresponding average masses of bound lipids are indicated below. Dashed and dotted lines are annotated with possible lipid compositions corresponding to no enrichment (1:1:1), PG enrichment (2:1:1), and PC enrichment (2:2.5:1). Grey shading indicates possible PC enrichment.

To compare our gas-phase results with solution-phase binding, we developed a new approach to rapidly extract AmtB-lipid complexes in solution rather than the gas phase. Previous studies show that the exchange between bound lipids and detergent molecules can be used to distinguish non-annular lipid interactions. ${ }^{26-27}$ Thus, we added detergents to break up the nanodisc and displace non- or weaklyinteracting lipids around AmtB. We tested tetraethylene glycol monooctyl ether (C8E4), Triton X-100, and lauryl dimethyl amine oxide (LDAO) because they require low collisional activation to dissociate from membrane proteins. ${ }^{28-29}$ Saccharide detergents are common for membrane protein solubilization, so we also tested octyl glucoside (OG).

To test their capacity for detergent extraction of AmtB from nanodiscs, different incubation times and detergent concentrations were screened. With C8E4 and Triton X-100, AmtB could easily be captured with both high and low numbers of bound lipids following minimal incubation times. Conversely, AmtB could only be captured with high numbers of bound lipids following incubation with LDAO and OG. Higher concentrations and longer incubation times helped with isolating lower numbers of bound lipids, but we continued with only C8E4 and Triton X-100. The final concentration of detergent was limited to $2 \times$ the critical micelle 

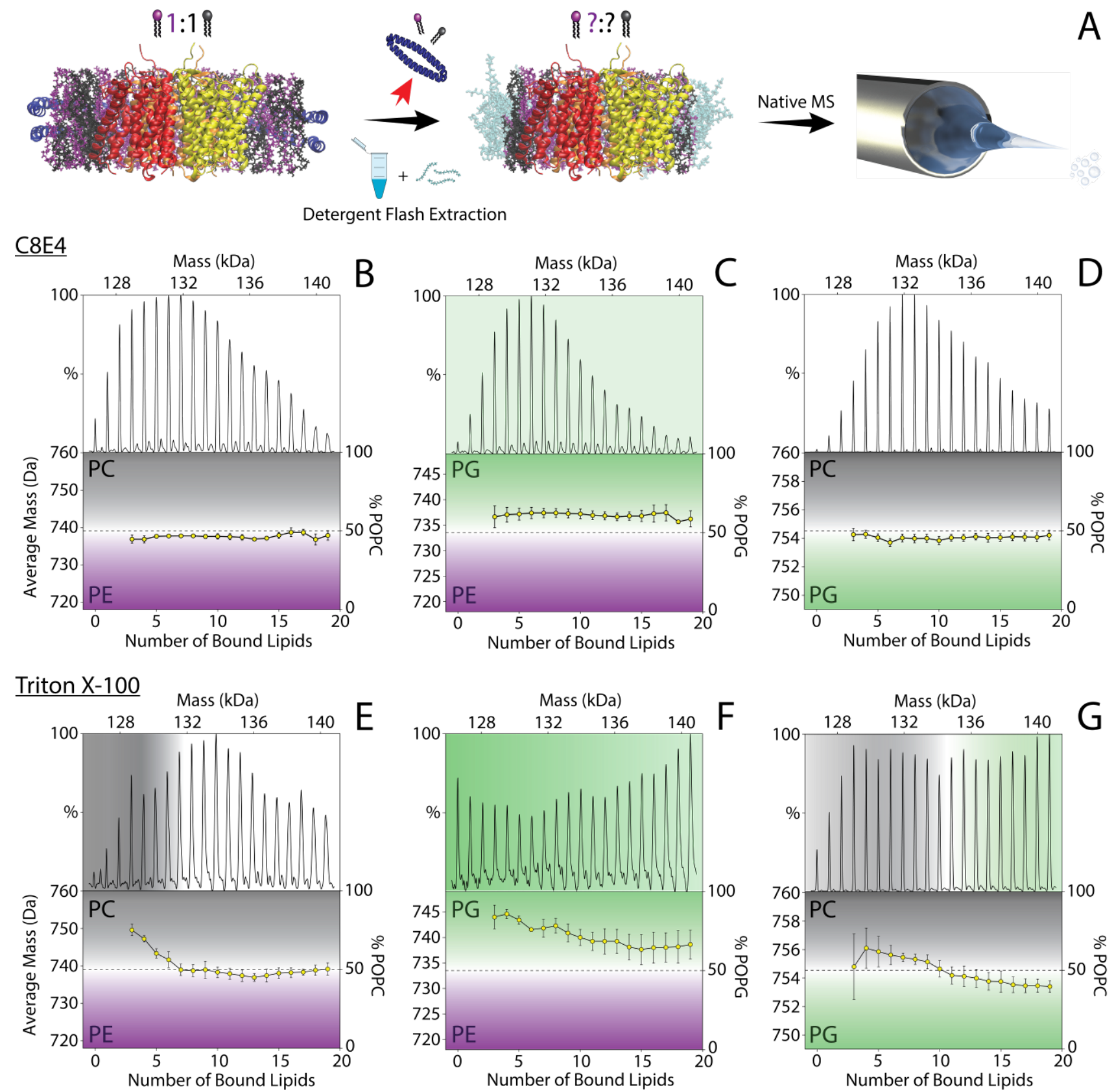

Figure 3. (A) Schematic for detergent flash extraction of AmtB-lipid complexes from binary lipid nanodiscs for native MS. Summed deconvolved mass spectra of AmtB-lipid complexes extracted with C8E4 (B-D) or Triton X-100 (E-G) from 50\% (B, E) PC:PE nanodiscs, (C, F) PG:PE nanodiscs, and (D, G) PC:PG nanodiscs. The corresponding average masses of bound lipids are plotted below the deconvolved mass spectra. Black, green, and purple regions represent enrichment in PC, PG, and PE, respectively. White regions represent no lipid enrichment. The initial expected average lipid masses corresponding to $50 \%$ lipid mixtures are indicated by dashed lines.

concentration (CMC) to improve mass spectra quality. Native MS revealed that AmtB is extracted into mixed lipid-detergent complexes devoid of MSP (Figure 3A).

With C8E4, AmtB flash-extracted from 50\% PC:PE nanodiscs had almost no enrichment (Figure 3B). AmtB from 50\% PG:PE was slightly enriched in PG but to a lesser degree than with the gas-phase ejection studies (Figure 3C). Finally, AmtB extracted from 50\% PC:PG also showed little to no enrichment (Figure 3D). Overall, the lipid selectivity of AmtB observed with gas-phase ejection was mostly absent when extracted into C8E4 detergent micelles. A slight preference for PG is retained, but selective binding of PC is completely lost.

We then probed lipid enrichment when flash extracting with Triton X-100. Here, AmtB extracted from 50\% PC:PE nanodiscs had significant PC enrichment for the five mosttightly bound lipids and no enrichment for the next 14 lipids (Figure 3E), very similar to results from gas-phase ejection. For 50\% PG:PE nanodiscs, all lipids were enriched in PG with lower numbers of lipids showing progressively more enrichment (Figure 3F). Finally, AmtB flash-extracted from 


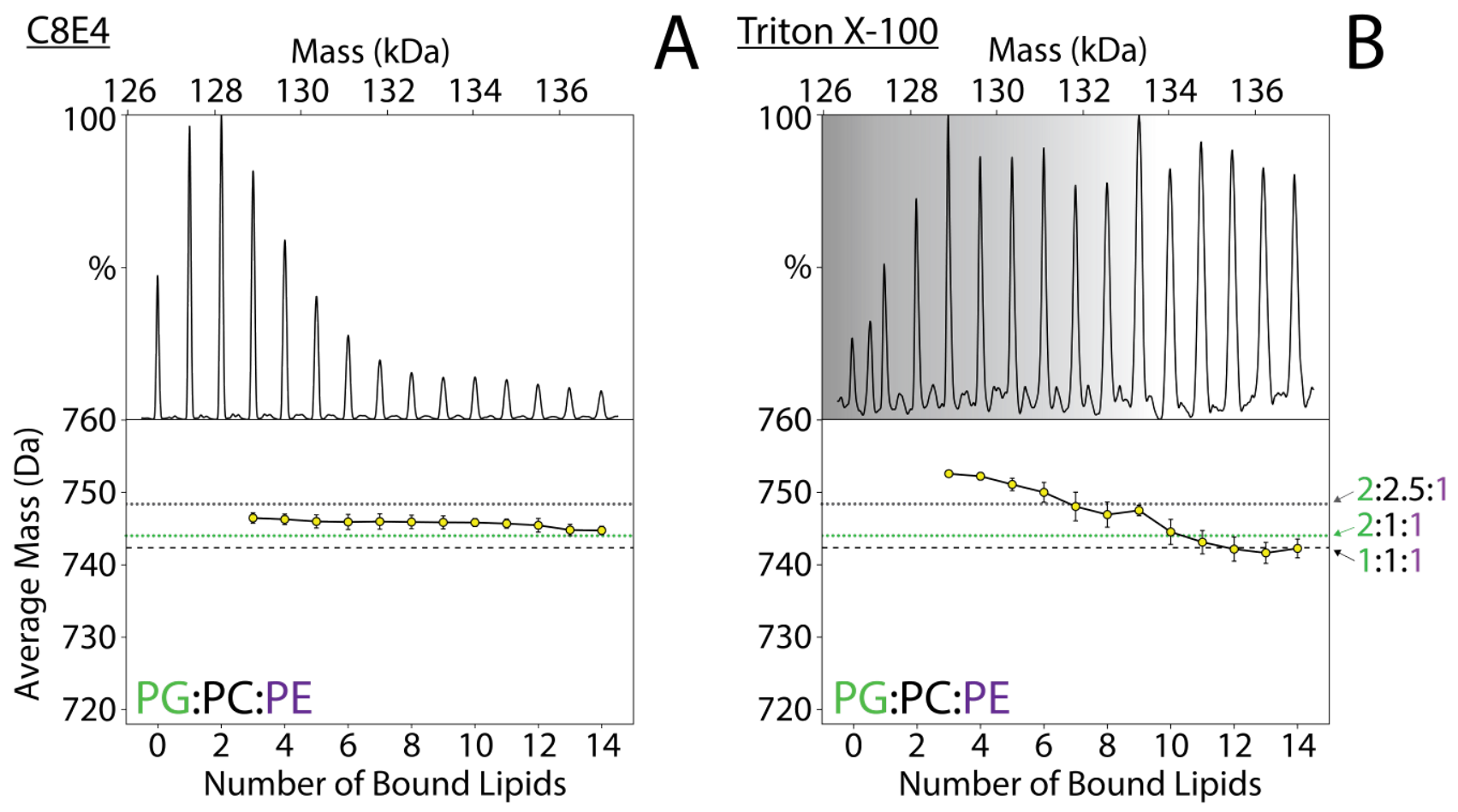

Figure 4. Summed deconvolved mass spectra of AmtB-lipid complexes extracted from 1:1:1 PG:PC:PE lipid nanodiscs using (A) C8E4 and (B) Triton X-100. The corresponding average masses of bound lipids are indicated below. Dashed and dotted lines are annotated with possible lipid compositions corresponding to no enrichment, PG enrichment, and PC enrichment. Grey shading indicates possible PC enrichment.

50\% PC:PG nanodiscs had distinct enrichment for two subsets of lipids (Figure 3G). The first subset of tightly bound lipids was enriched in PC, and the less tightly bound subset was enriched in PG. In all three cases, Triton X-100 extraction matched the lipid selectivity observed with gas-phase ejection.

Finally, we extracted AmtB from 1:1:1 PG:PC:PE nanodiscs. With C8E4, all lipids showed a similar average lipid mass, consistent with a constant lipid enrichment in PG but a loss of selective PC binding for tightly bound lipids (Figure 4A). For Triton X-100, lipids showed a gradually heavier average mass for more tightly bound lipids, indicating likely PC enrichment for lower numbers of bound lipids (Figure 4B). Thus, the lipid selectivity for AmtB flash-extracted from ternary nanodiscs with Triton X-100 qualitatively agrees with both the Triton X-100 dataset from binary nanodiscs and with gas-phase ejection from nanodiscs. Together, these results indicate a broad selectivity for PG lipids, a few tight binding sites for PC, and limited preference for PE.

The differences observed between C8E4 and Triton X100 reinforce that there is a delicate balance for detergents between compatibility with native MS, solubilization effectiveness, and disruption of lipid interactions. These factors should be taken into careful consideration when selecting detergents for extracting membrane proteins. An interesting approach would be to use novel oligoglycerol detergents $^{30}$ to fine-tune the propensity for delipidation to study different types of protein-lipid interactions.

\section{CONCLUSIONS}

Mounting evidence is revealing the vital role of lipids in membrane protein structure and function. ${ }^{13,31-34}$ High-resolution biophysical techniques can provide detailed structures of membrane proteins in stable complexes with nonannular lipids. However, many lipid interactions are transient and heterogeneous, and it is challenging to measure how membrane proteins selectively remodel their surrounding lipid environment to bind these annular lipids. Here, we demonstrated that native MS can be used to probe lipid remodeling by membrane proteins in heterogeneous lipid nanodiscs and distinguish lipid selectivity for a larger number of bound lipids.

Gas-phase ejection and solution-phase detergent flash extraction for native MS provide orthogonal approaches for investigating how AmtB remodels its local lipid environment in two- and three-component nanodiscs. Both approaches reveal that AmtB is broadly enriched in bound PG, has a few tightly bound PC lipids, but shows no selective enrichment in PE.

Detergent flash extraction provides a quick, simple, and complementary approach for investigating membrane protein-lipid interactions but requires careful selection of detergents. Triton X-100 extraction preserved enrichment of PC and PG lipids, but C8E4 extraction showed little to no enrichment, demonstrating that detergents can selectively alter membrane protein-lipid interactions. Overall, these two approaches are broadly applicable for distinguishing and quantifying membrane protein-lipid selectivity, providing detailed insights into the biophysics of membrane proteinlipid interactions in heterogeneous lipid bilayers.

ASSOCIATED CONTENT 

available under aCC-BY-NC-ND 4.0 International license.

Supporting Information. Native mass spectra for AmtB nanodiscs. This material is available free of charge via the Internet at http://pubs.acs.org.

\section{AUTHOR INFORMATION}

\section{Corresponding Author}

*mtmarty@arizona.edu

Notes

The authors declare no competing financial interest.

\section{ACKNOWLEDGMENTS}

The authors thank Maria Reinhardt-Szyba, Kyle Fort, and Alexander Makarov at Thermo Fisher Scientific for support on the Q-Exactive HF UHMR instrument. The pMSP1E3D1 plasmid was a gift from Stephen Sligar (Addgene plasmid no. 20066). This work was funded by the National Institute of General Medical Sciences and National Institutes of Health (Grant R35 GM128624). The content is solely the responsibility of the authors and does not necessarily represent the official views of the NIH.

\section{REFERENCES}

1. Overington, J.; Al-Lazikani, B.; Hopkins, A., How many drug targets are there? Nature Reviews Drug Discovery 2006, 5, 993-996.

2. Krogh, A.; Larsson, B.; von Heijne, G.; Sonnhammer, E. L., Predicting transmembrane protein topology with a hidden Markov model: application to complete genomes. J Mol Biol 2001, 305 (3), 567-80.

3. Almen, M. S.; Nordstrom, K. J.; Fredriksson, R.; Schioth, H. B., Mapping the human membrane proteome: a majority of the human membrane proteins can be classified according to function and evolutionary origin. BMC Biol 2009, 7, 50.

4. Brown, M. F., Soft Matter in Lipid-Protein Interactions. Annu Rev Biophys 2017, 46, 379-410.

5. Dowhan, W.; Bogdanov, M., Lipid-protein interactions as determinants of membrane protein structure and function. Biochem Soc Trans 2011, 39 (3), 767-74.

6. McMahon, H. T.; Gallop, J. L., Membrane curvature and mechanisms of dynamic cell membrane remodelling. Nature 2005, 438 (7068), 590-6.

7. Welch, L. G.; Munro, S., A tale of short tails, through thick and thin: investigating the sorting mechanisms of Golgi enzymes. FEBS Lett 2019, 593 (17), 2452-2465.

8. Laganowsky, A.; Reading, E.; Allison, T. M.; Ulmschneider, M. B.; Degiacomi, M. T.; Baldwin, A. J.; Robinson, C. V., Membrane proteins bind lipids selectively to modulate their structure and function. Nature 2014, 510 (7503), 172-175.

9. Landreh, M.; Marty, M. T.; Gault, J.; Robinson, C. V., A sliding selectivity scale for lipid binding to membrane proteins. Curr Opin Struct Biol 2016, 39, 54-60.

10. Muller, M. P.; Jiang, T.; Sun, C.; Lihan, M.; Pant, S.; Mahinthichaichan, P.; Trifan, A.; Tajkhorshid, E., Characterization of Lipid-Protein Interactions and Lipid-Mediated Modulation of Membrane Protein Function through Molecular Simulation. Chem Rev 2019, 119 (9), 6086-6161.

11. Bagheri, Y.; Ali, A. A.; You, M., Current Methods for Detecting Cell Membrane Transient Interactions. Front Chem 2020, 8, 603259.

12. Gupta, K.; Li, J.; Liko, I.; Gault, J.; Bechara, C.; Wu, D.; Hopper, J. T. S.; Giles, K.; Benesch, J. L. P.; Robinson, C. V., Identifying key membrane protein lipid interactions using mass spectrometry. Nat Protoc 2018, 13 (5), 1106-1120.

13. Gupta, K.; Donlan, J. A. C.; Hopper, J. T. S.; Uzdavinys, P.; Landreh, M.; Struwe, W. B.; Drew, D.; Baldwin, A. J.; Stansfeld, P. J.; Robinson, C. V., The role of interfacial lipids in stabilizing membrane protein oligomers. Nature 2017, 541 (7637), 421-424.
14. Patrick, J. W.; Boone, C. D.; Liu, W.; Conover, G. M.; Liu, Y.; Cong, X.; Laganowsky, A., Allostery revealed within lipid binding events to membrane proteins. Proc Natl Acad Sci U S A 2018, 115 (12), 2976-2981.

15. Bechara, C.; Robinson, C. V., Different modes of lipid binding to membrane proteins probed by mass spectrometry. J Am Chem Soc 2015, 137 (16), 5240-7.

16. Keener, J. E.; Zambrano, D. E.; Zhang, G.; Zak, C. K.; Reid, D. J.; Deodhar, B. S.; Pemberton, J. E.; Prell, J. S.; Marty, M. T., Chemical Additives Enable Native Mass Spectrometry Measurement of Membrane Protein Oligomeric State within Intact Nanodiscs. J Am Chem Soc 2019, 141 (2), 1054-1061.

17. Zhang, G.; Keener, J. E.; Marty, M. T., Measuring Remodeling of the Lipid Environment Surrounding Membrane Proteins with Lipid Exchange and Native Mass Spectrometry. Anal Chem 2020, 92 (8), 5666-5669.

18. Mirandela, G. D.; Tamburrino, G.; Hoskisson, P. A.; Zachariae, U.; Javelle, A., The lipid environment determines the activity of the Escherichia coli ammonium transporter AmtB. FASEB J 2019, 33 (2), 1989-1999.

19. Reid, D. J.; Keener, J. E.; Wheeler, A. P.; Zambrano, D. E.; Diesing, J. M.; Reinhardt-Szyba, M.; Makarov, A.; Marty, M. T., Engineering Nanodisc Scaffold Proteins for Native Mass Spectrometry. Anal Chem 2017, 89 (21), 11189-11192.

20. Ritchie, T. K.; Grinkova, Y. V.; Bayburt, T. H.; Denisov, I. G.; Zolnerciks, J. K.; Atkins, W. M.; Sligar, S. G., Chapter 11 Reconstitution of membrane proteins in phospholipid bilayer nanodiscs. Methods Enzymol 2009, 464, 211-31.

21. van de Waterbeemd, M.; Fort, K. L.; Boll, D.; ReinhardtSzyba, M.; Routh, A.; Makarov, A.; Heck, A. J., High-fidelity mass analysis unveils heterogeneity in intact ribosomal particles. Nat Methods 2017, 14 (3), 283-286.

22. Marty, M. T.; Baldwin, A. J.; Marklund, E. G.; Hochberg, G. K.; Benesch, J. L.; Robinson, C. V., Bayesian deconvolution of mass and ion mobility spectra: from binary interactions to polydisperse ensembles. Anal Chem 2015, 87 (8), 4370-6.

23. Reid, D. J.; Diesing, J. M.; Miller, M. A.; Perry, S. M.; Wales, J. A.; Montfort, W. R.; Marty, M. T., MetaUniDec: High-Throughput Deconvolution of Native Mass Spectra. J Am Soc Mass Spectrom 2019, 30 (1), 118-127.

24. Donor, M. T.; Wilson, J. W.; Shepherd, S. O.; Prell, J. S., Lipid head group adduction to soluble proteins follows gas-phase basicity predictions: Dissociation barriers and charge abstraction. International Journal of Mass Spectrometry 2021, 469.

25. Cong, X.; Liu, Y.; Liu, W.; Liang, X.; Laganowsky, A., Allosteric modulation of protein-protein interactions by individual lipid binding events. Nat Commun 2017, 8 (1), 2203.

26. Bolla, J. R.; Corey, R. A.; Sahin, C.; Gault, J.; Hummer, A.; Hopper, J. T. S.; Lane, D. P.; Drew, D.; Allison, T. M.; Stansfeld, P. J.; Robinson, C. V.; Landreh, M., A Mass-Spectrometry-Based Approach to Distinguish Annular and Specific Lipid Binding to Membrane Proteins. Angew Chem Int Ed Engl 2020, 59 (9), 35233528.

27. Fiorentino, F.; Sauer, J. B.; Qiu, X.; Corey, R. A.; Cassidy, C. K.; Mynors-Wallis, B.; Mehmood, S.; Bolla, J. R.; Stansfeld, P. J.; Robinson, C. V., Dynamics of an LPS translocon induced by substrate and an antimicrobial peptide. Nat Chem Biol 2021, 17 (2), 187-195.

28. Konijnenberg, A.; Yilmaz, D.; Ingolfsson, H. I.; Dimitrova, A.; Marrink, S. J.; Li, Z.; Venien-Bryan, C.; Sobott, F.; Kocer, A., Global structural changes of an ion channel during its gating are followed by ion mobility mass spectrometry. Proc Natl Acad Sci U S A 2014, 111 (48), 17170-5.

29. Reading, E.; Liko, I.; Allison, T. M.; Benesch, J. L.; Laganowsky, A.; Robinson, C. V., The role of the detergent micelle in preserving the structure of membrane proteins in the gas phase. Angew Chem Int Ed Engl 2015, 54 (15), 4577-81.

30. Urner, L. H.; Liko, I.; Yen, H. Y.; Hoi, K. K.; Bolla, J. R.; Gault, J.; Almeida, F. G.; Schweder, M. P.; Shutin, D.; Ehrmann, S.; Haag, R.; Robinson, C. V.; Pagel, K., Modular detergents tailor the purification 
bioRxiv preprint doi: https://doi.org/10.1101/2021.08.17.456692; this version posted August 17, 2021. The copyright holder for this preprint (which was not certified by peer review) is the author/funder, who has granted bioRxiv a license to display the preprint in perpetuity. It is made available under aCC-BY-NC-ND 4.0 International license.

and structural analysis of membrane proteins including G-protein coupled receptors. Nat Commun 2020, 11 (1), 564.

31. McDowell, M. A.; Heimes, M.; Fiorentino, F.; Mehmood, S.; Farkas, A.; Coy-Vergara, J.; Wu, D.; Bolla, J. R.; Schmid, V.; Heinze, R.; Wild, K.; Flemming, D.; Pfeffer, S.; Schwappach, B.; Robinson, C. V.; Sinning, I., Structural Basis of Tail-Anchored Membrane Protein Biogenesis by the GET Insertase Complex. Mol Cell 2020, 80 (1), $72-86$ e 7.

32. Yen, H. Y.; Hoi, K. K.; Liko, I.; Hedger, G.; Horrell, M. R.; Song, W.; Wu, D.; Heine, P.; Warne, T.; Lee, Y.; Carpenter, B.; Pluckthun, A.; Tate, C. G.; Sansom, M. S. P.; Robinson, C. V., PtdIns(4,5)P2 stabilizes active states of GPCRs and enhances selectivity of G-protein coupling. Nature 2018, 559 (7714), 423427.

33. Pyle, E.; Kalli, A. C.; Amillis, S.; Hall, Z.; Lau, A. M.; Hanyaloglu, A. C.; Diallinas, G.; Byrne, B.; Politis, A., Structural Lipids Enable the Formation of Functional Oligomers of the Eukaryotic Purine Symporter UapA. Cell Chem Biol 2018, 25 (7), 840-848 e4.

34. Debruycker, V.; Hutchin, A.; Masureel, M.; Ficici, E.; Martens, C.; Legrand, P.; Stein, R. A.; McHaourab, H. S.; FaraldoGomez, J. D.; Remaut, H.; Govaerts, C., An embedded lipid in the multidrug transporter LmrP suggests a mechanism for polyspecificity. Nat Struct Mol Biol 2020, 27 (9), 829-835.

For Table of Contents Only:

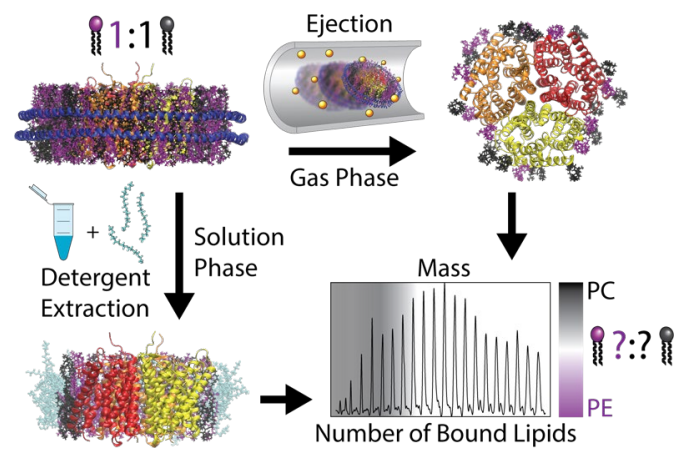

